\title{
Proton Pump Inhibitor Switching Strategy after Failure of Standard Triple Therapy for Helicobacter pylori Infection
}

\author{
Jaeyoung Kim, Yeon-Ji Kim, Woo Chul Chung \\ Department of Internal Medicine, St. Vincent's Hospital, College of Medicine, The Catholic University of Korea, Suwon, Korea
}

\begin{abstract}
Background/Aims: It is still unknown whether cytochrome P450 (CYP) 2C19 polymorphisms influence Helicobacter pylori (H. pylori) eradication, especially in eastern Asia. We aimed to evaluate how changes in proton pump inhibitor (PPI) strategies could be used to overcome the effects of CYP2C19 polymorphism on $H$. pylori eradication rate when it is used as the second-line regimen after the failure of standard triple therapy.

Materials and Methods: We performed a retrospective observation study of 675 patients in whom standard triple therapy for $H$. pylori infection was not effective between January 2009 to December 2018. All patients underwent a classic bismuth-containing quadruple therapy (10 to 14-day regimen), and their eradication rates were evaluated for several years. We compared the eradication rates in patients with or without the second-line PPI switch. Further, we assessed differences in eradication rates with or without the strategy using esomeprazole and rabeprazole, which are not influenced significantly by CYP2C19 genetic polymorphism.

Results: The eradication rate was $81.0 \%$ in individuals who received the second-line PPI switch, but it was $74.8 \%$ without switching $(P=0.14)$. In the strategy using esomeprazole and rabeprazole, the eradication rate was $84.6 \%$, compared to $76.5 \%$ in the control group $(P=0.03)$. Finally, in the group of patients who switched to rabeprazole, the eradication rates were $85.6 \%$, compared to $77.6 \%$ in the group who switched to pantoprazole $(P=0.05)$.

Conclusions: Switching to PPI, which is not influenced by CYP2C19 genetic polymorphism, increases the efficiency of eradication after the failure of standard triple therapy. (Korean J Helicobacter Up Gastrointest Res 2020;20:146-152)
\end{abstract}

Key Words: CYP2C19 protein, human; Disease eradication; Helicobacter pylori; Proton pump inhibitors

\section{INTRODUCTION}

Helicobacter pylori (H. pylori) affects nearly half of the world's population and is a pathogen prevalent in gastrointestinal disorders. ${ }^{1}$ The eradication of $H$. pylori in gastrointestinal diseases is important for the treatment of diseases and recurrence prevention and is highly related to adverse outcomes. ${ }^{2,3}$ In addition, the eradication of $H . p y^{-}$ lori could be the most efficient method to prevent gastric cancer in patients with mild or no mucosal atrophy. ${ }^{4-6}$ Furthermore, the indications for the eradication of $H . p y^{-}$ lori were extended to $H$. pylori-positive functional dyspepsia according to the Kyoto and ROME IV criteria, and this is thought to be related to the increased use of eradication therapy. ${ }^{7,8}$

Patients with $H$. pylori-associated atrophic gastritis can

Received: September 27, 2019 Revised: October 28, 2019 Accepted: November 12, 2019 Corresponding author: Yeon-Ji Kim

Department of Internal Medicine, St. Vincent's Hospital, College of Medicine, The Catholic University of Korea, 93 Jungbu-daero, Paldal-gu, Suwon 16247, Korea Tel: +82-31-881-8582, Fax: +82-31-253-8898, E-mail: dr.kimyj@gmail.com receive eradication therapy covered by Korean Health Insurance, a universal coverage system, so the number of patients undergoing eradication therapy has increased in many clinical practices. The eradication rate of $H$. pylori infections using first-line standard triple therapy has decreased over the last 20 years, and antibiotic resistance and poor compliance are thought to be the main causes of the failure of standard triple therapy. Based on the Maastricht IV Consensus Reports, bismuth-based classic quadruple therapy comprised of a proton pump inhibitor (PPI), bismuth, metronidazole, and tetracycline has been applied worldwide in $H$. pylori second-line rescue therapy. However, accumulating data and meta-analysis showed that the eradication rate from bismuth-based classic quadruple therapy as the second-line treatment was only $78 \%{ }^{10}$ PPIs are mainly metabolized by the cytochrome P450 (CYP) system, in which CYP2C19 is the principal enzyme and CYP3A4 also contributes to a lesser degree. CYP2C19 genetic polymorphisms are considered another issue in the failure of standard triple therapy for $H$. pylori infections. Three CYP2C19 genetic polymorphisms have been re-

Copyright $\odot 2020$ Korean College of Helicobacter and Upper Gastrointestinal Research

@) The Korean Journal of Helicobacter and Upper Gastrointestinal Research is an Open-Access Journal. All articles are distributed under the terms of the Creative Commons Attribution Non-Commercial License (http:// creativecommons.org/licenses/by-nc/4.0) which permits unrestricted non-commercial use, distribution, and reproduction in any medium, provided the original work is properly cited. 
ported, homozygous extensive metabolizers, heterozygous extensive metabolizers, and poor metabolizers (PM), by the combination of wild or mutant alleles. ${ }^{11,12}$

A meta-analysis of the effect of CYP2C19 genetic polymorphisms on the efficacy of PPI-based triple therapy showed higher $H$. pylori eradication rates in PMs compared to homozygous extensive metabolizers. ${ }^{13}$ Rabeprazole is characterized by non-enzymatic metabolism with renal elimination of the metabolites, and esomeprazole is mainly metabolized by CYP3A4. In general, rabeprazole or esomeprazole therapy had no significant effect on $H$. pylori eradication rates among patients with the three CYP2C19 genetic polymorphisms. ${ }^{14,15}$ According to studies conducted in Asia, including Japan, China, and Korea, the effects of CYP2C19 genetic polymorphisms were not clear, and the efficacy of PPI-based standard triple therapy was not affected by CYP2C19 genetic polymorphisms. ${ }^{16-19}$ Furthermore, CYP2C19 genotypes did not show obvious impacts on other rescue therapies. In this study, we aimed to evaluate the clinical impact of changing the PPI in the second-line eradication regimen and analyze the effect of the strategy using esomeprazole and rabeprazole, which are not influenced significantly by CYP2C19 genetic polymorphisms.

\section{MATERIALS AND METHODS}

\section{Study design}

The study was performed in accordance with the Declaration of Helsinki and approved by the Ethics Committee of the Catholic University of Korea (VC19REST0133). The retrospective observational study included patients with peptic ulcers or atrophic gastritis who had previously been treated unsuccessfully with standard triple therapy for $H$. pylori eradication from January 2009 to December 2018 at St.Vincent's Hospital, The Catholic University of Korea.

The eligibility criteria for this study included patients over 18 years of age with gastric $H$. pylori infections. All patients in this study received standard triple therapy with a regimen that consisted of amoxicillin $1.0 \mathrm{~g}$ b.i.d. and clarithromycin $500 \mathrm{mg}$ b.i.d. with various kinds of PPIs for 14 days, which failed to eradicate the infection, confirmed by positive ${ }^{13} \mathrm{C}$-urea breath test (UBiT-IR 300;
Photal OTSUKA Electronics, Osaka, Japan) results after 4 6 weeks of treatment. Patients with previous gastric surgery, chronic illness (renal disease: serum creatinine $>1.5 \mathrm{mg} / \mathrm{dL}$, heart failure: ejection fraction <20\%) or pregnancy were excluded from this study due to possible effects on drug metabolism.

A classic bismuth-containing quadruple regimen was used as the second-line therapy, consisting of PPIs (pantoprazole $40 \mathrm{mg}$, lansoprazole $30 \mathrm{mg}$, esomeprazole $20 \mathrm{mg}$, or rabeprazole $20 \mathrm{mg}$ ) b.i.d., tripotassium dicitrate bismuthate (DENOL Greencross Co., Seoul, Korea) 300 mg q.i.d., metronidazole $500 \mathrm{mg}$ t.i.d., and tetracycline $500 \mathrm{mg}$ q.i.d. for 10 14 days.

Successful eradication was assessed by the ${ }^{13} \mathrm{C}$-urea breath test conducted 4 6 weeks after the completion of treatment. The ${ }^{13} \mathrm{C}$-urea breath test was performed with a pranactin-citric drug product, a component of the BreathTek UBT Kit (Korea Otsuka Pharmaceutical Co. Ltd., Seoul, Korea). Three grams of reconstituted panactin-citric containing $75 \mathrm{mg}$ of ${ }^{13} \mathrm{C}$-urea was ingested by the patient. Breath samples before and 20 minutes after the administration of ${ }^{13} \mathrm{C}$-urea were collected after a mouthwash. The ${ }^{13} \mathrm{C} /{ }^{12} \mathrm{C}$ ratio in the breath samples was measured by an infrared spectrophotometer (UBiT-IR300; Korea Otsuka Pharmaceutical Co. Ltd.). The changes in the ${ }^{13} \mathrm{C}$ value over baseline were expressed as $\Delta^{13} \mathrm{C}$. A positive result was defined as an increase of $>2.5 \%$. First, we evaluated the eradication rates with bismuth-based classic quadruple therapy as the second-line treatment between the first five years (2009 2013) and the last five years (2014 2018). Second, we compared the eradication rate with or without second-line PPI substitutions in the second-line eradication regimen, and also examined the difference with or without the strategy using esomeprazole and rabeprazole, which were not influenced significantly by CYP2C19 genetic polymorphisms.

\section{Statistics}

All data were recorded on standard forms and computer-analyzed. Student's t-test was used to compare the continuous variables between the two groups. Differences between the dichotomous variables were evaluated by the 
chi-squared test. The trends in the annual differences were evaluated by chi-squared tests. The calculations were performed with SPSS package software (SPSS version 25.0; SPSS Inc., Chicago, IL, USA). $P$-values of less than 0.05 were considered significant.

\section{RESULTS}

A total of 675 patients with failed first-line eradication received second-line bismuth-containing quadruple therapy from 2009 to 2018 (Fig. 1). The total eradication rate was $80.4 \%(543 / 675)$, and the number of first-line erad-

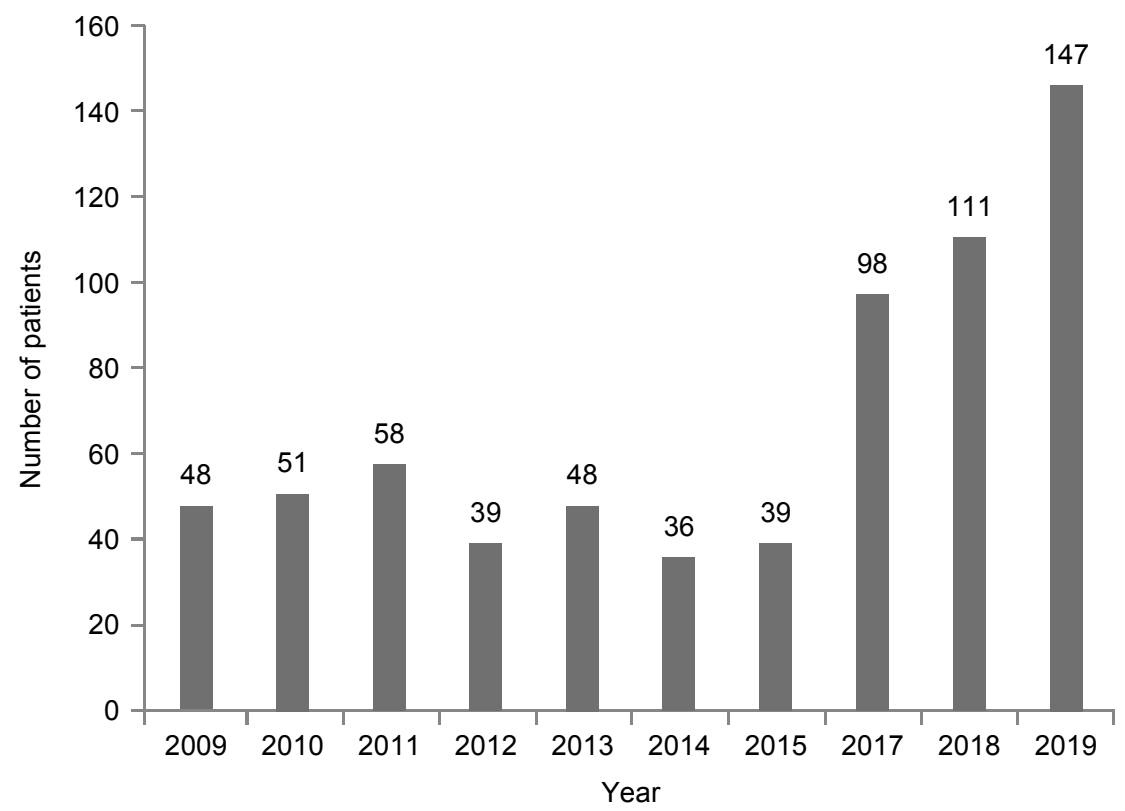

Fig. 1. Number of patients receiving the second-line bismuth containing quadruple therapy after the failure of first-line eradication (proton pump inhibitor-clarithromycin-amoxicillin triple therapy) from 2009 to 2018. A total of 675 patients are analyzed.

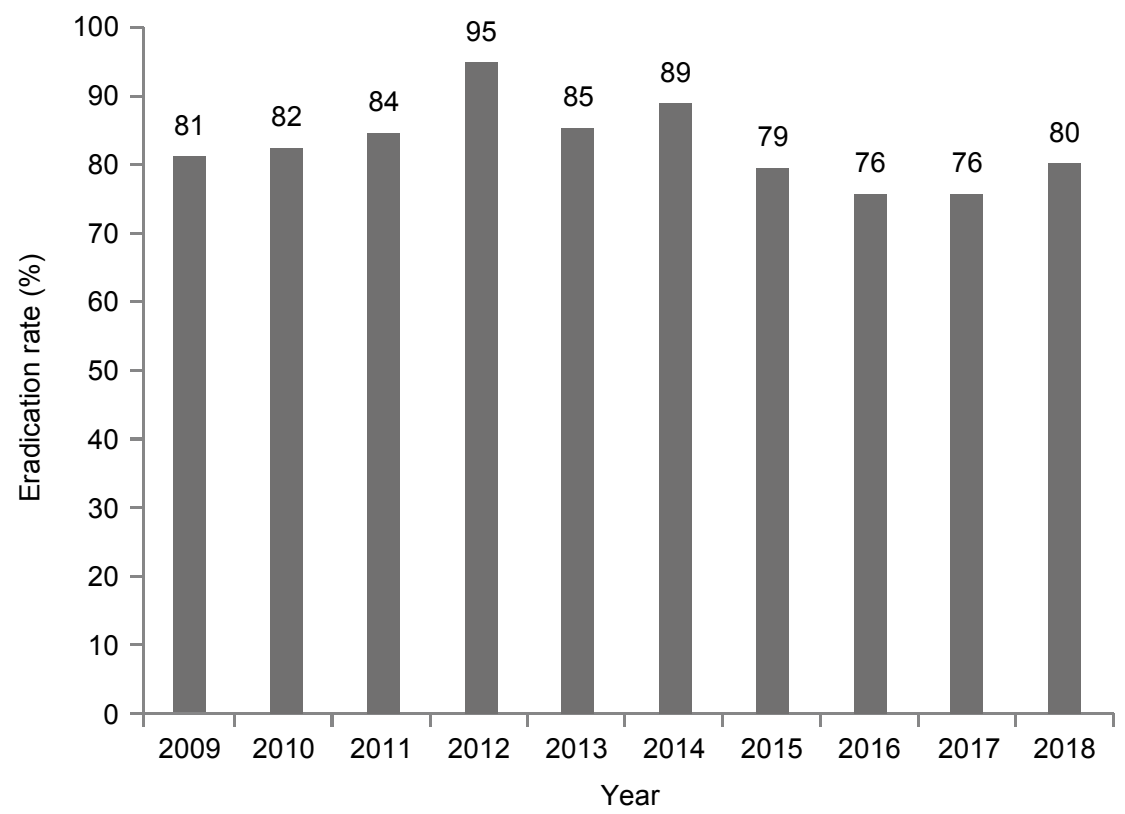

Fig. 2. Eradication rates (\%) of bismuth containing quadruple therapy as the second-line regimen from 2009 to 2018 . The eradication rate is $85.2 \%$ $(204 / 244)$ for the first 5 years, and $78.7 \%$ (339/431) for the last 5 years. There is a significantly decreasing tendency in the eradication rates $(P=0.03)$. 
ication failures rapidly increased since 2016. For the first five years (2009 2013), the eradication rate was $85.2 \%$ (204/244), whereas it was $78.7 \%$ (339/431) for the last five years (2014 2018). There was a significantly decreasing tendency in the eradication rates $(P=0.03)$ (Fig. 2).

Several kinds of PPIs, including omeprazole, pantoprazole, lansoprazole, esomeprazole, and rabeprazole, were used from 2013 to 2018. Between January 2013 and December 2018, a total of 479 patients received a bismuth-based quadruple regimen as the second-line therapy, and $H$. pylori was successfully eradicated in $380 \mathrm{pa-}$ tients, 181 males and 199 females (mean age, 59.1 11.9 years). In the second-line eradication therapy failure group, 99 patients (49 males and 50 females; mean age, $58.8 \pm 11.8$ years) were evaluated (Table 1$)$. Various kinds of PPIs were chosen in the first and second-line regimens for $H$. pylori eradication (Fig. 3). The patients with different choices of PPIs between the first and second-line regimens were referred to as the PPI-switching group, with an eradication rate was $73.5 \%$ (352/479). The eradication rate was $81.0 \%(285 / 352)$ in the second-line
PPI-switching group, and $74.8 \%$ without switching (95/127) $(P=0.14)$. When we defined a strategy to overcome CYP2C19 polymorphism according to the use of esomeprazole and rabeprazole, which were are influenced significantly by CYP2C19 genetic polymorphism, the frequency with the strategy was 35.3\% (169/479). The eradication rate was $84.6 \%(143 / 169)$ with the strategy, whereas it was $76.5 \%$ (237/310) without the strategy. The

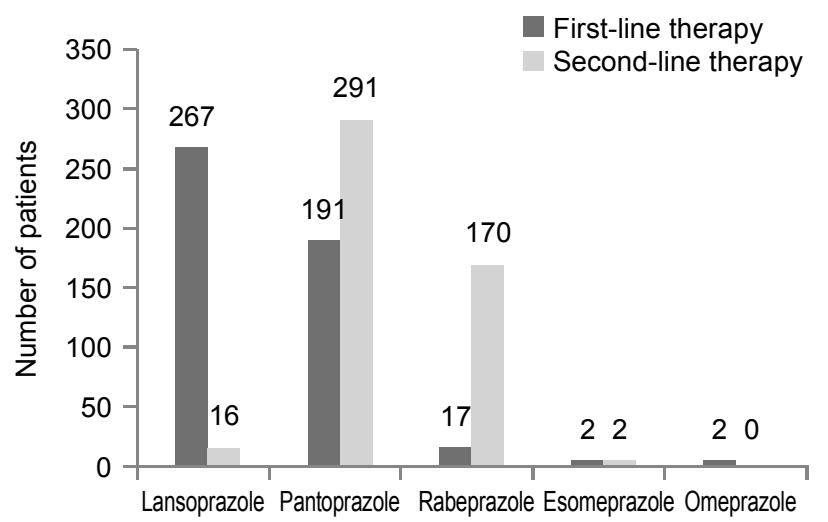

Fig. 3. Choice of proton pump inhibitors in the first and second-line regimens for Helicobacter pylori eradication.

Table 1. Clinical Characteristics in Patients Who Received Successful or Unsuccessful Second-line Bismuth Containing Quadruple Therapy

\begin{tabular}{|c|c|c|c|}
\hline & Success $(n=380)$ & Failure $(n=99)$ & $P$-value \\
\hline Sex (male:female) & 181:199 & 49:50 & 0.74 \\
\hline Age (years) & $59.1 \pm 11.9$ & $58.8 \pm 11.8$ & 0.84 \\
\hline Current smoker & $68(18)$ & $21(21)$ & 0.45 \\
\hline Alcohol drinking & $93(24)$ & $28(28)$ & 0.44 \\
\hline Diagnosis & & & $0.03^{\mathrm{a}}$ \\
\hline Peptic ulcer disease & $162(43)$ & $48(48)$ & \\
\hline Gastric ulcer & $90(24)$ & $20(20)$ & \\
\hline Duodenal ulcer & $72(19)$ & $28(28)$ & \\
\hline Atrophic gastritis & $177(47)$ & $33(33)$ & \\
\hline Gastric epithelial dysplasia & $41(11)$ & $18(18)$ & \\
\hline Ten days regimen & $81(21)$ & $19(19)$ & 0.64 \\
\hline PPI change & $285(75)$ & $67(67)$ & 0.14 \\
\hline Using lansoprazole & $10(2)$ & $3(3)$ & \\
\hline Using pantoprazole & $132(34)$ & $38(38)$ & 0.14 \\
\hline Strategy to overcome $2 \mathrm{C} 19$ polymrphism & $143(38)$ & $26(26)$ & $0.03^{\mathrm{a}}$ \\
\hline Using esomeprazole & $0(0)$ & $2(2)$ & \\
\hline Using rabeprazole & $143(38)$ & $24(24)$ & \\
\hline
\end{tabular}

Values are presented as mean \pm standard deviation or $\mathrm{n}(\%)$.

PPI, proton pump inhibitor.

${ }^{a}$ Indicates statistical significance, $P<0.05$. 


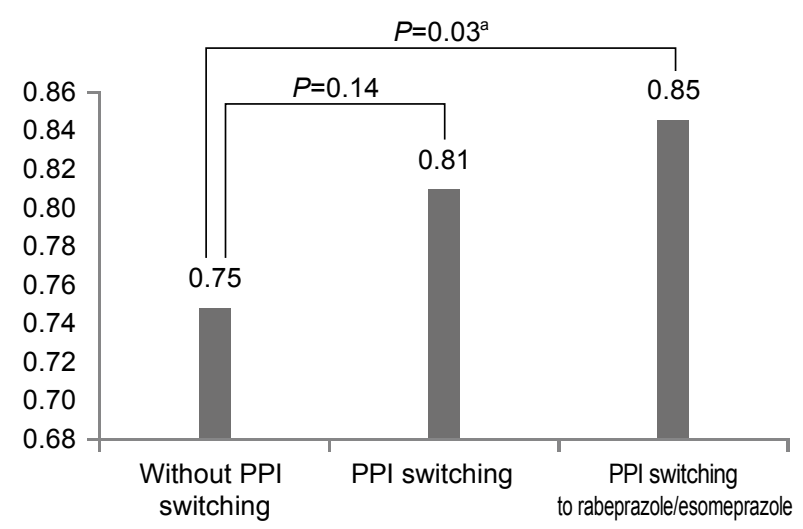

Fig. 4. Eradication rate with or without the second-line proton pump inhibitor (PPI) switching strategy. PPI switch to rabeprazole or esomeprazole shows an eradication rate of $84.6 \%$. The difference is statistically significant $(P=0.03) .{ }^{a}$ Indicates statistical significance, $P<0.05$.

difference between using such a strategy and not using was statistically significant ( $P=0.03)$ (Fig. 4). The majority of the patients in the PPI-switching group were changed to pantoprazole $(n=170)$ or rabeprazole $(n=167)$. In a comparison of the eradication rates using the two PPIs, the eradication rate of $H$. pylori infections in the rabeprazole switching group was $85.6 \%$ (143/167), whereas it was $77.6 \%(132 / 170)$ in the pantoprazole switching group (Fig. 5).

\section{DISCUSSION}

The more frequently eradication therapies are undertaken, the more frequently resistant strains of $H$. pylori appear. Now, the use of second-line rescue therapy is increasing because of the limited efficacy of initial PPI-based standard triple therapy. Although the most concerning factor in eradication failure is antibiotic resistance, especially to clarithromycin and metronidazole, the role of PPIs in the eradication of $H$. pylori cannot be ignored. PPIs play a pivotal role in the eradication of $H$. pylori infection. ${ }^{20}$ PPIs have a potent effect on concentrating antibiotics via suppressing gastric juice. Moreover, they have a weak anti-bacterial effect against $H$. pylori in $v^{-}$ tro, anti-urease activity, and anti-ATPase activity. ${ }^{21}$ In general, antibiotics could have a maximal antibacterial effect on microbial growth-dependent manner, and $H$. $p y^{-}$

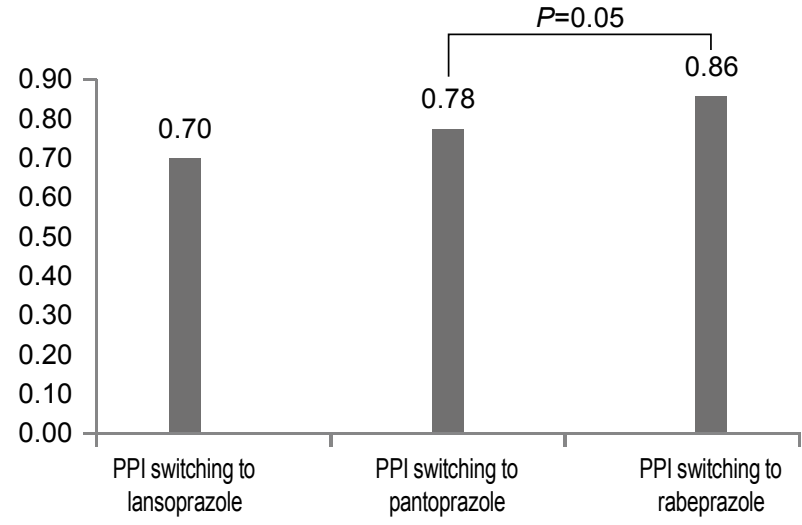

Fig. 5. Within the proton pump inhibitor (PPI) switching group, the eradication rate in the group who switched to rabeprazole was $85.6 \%$, while it was $77.6 \%$ in the group who switched to pantoprazole $(P=0.05)$.

lori growth can be found in the weakly acidic environment produced by PPIs. ${ }^{22}$ Additionally, delayed emptying and thinning of the mucosal layer induced by PPIs would make H. pylori vulnerable to antibiotics. ${ }^{23}$ The results of this study showed that the successful eradication rate decreased in second-line H. pylori eradication therapy despite the quantitative increase in the number of patients undergoing second-line therapy in the last five years. In clinical practice, increases in antibiotic resistance make clinicians scrutinize the eradication rate of the second-line rescue therapy for $H$. pylori infections. The Maastricht IV Consensus recommended bismuth-containing quadruple therapy (PPI+bismuth+metronidazole+tetracycline) as the second-line treatment option. ${ }^{9}$ In a recent meta-analysis, the mean eradication rate with the same regimen was only $78 \%{ }^{10}$

Unfortunately, the eradication rate decreased after the expansion of the indications for $H$. pylori eradication therapy. To identify better rescue eradication strategies, clinicians should consider multiple factors, such as antibiotic resistance, poor compliance, and more powerful suppression of gastric acid. When it comes to the suppression induced by PPIs, individual variability could be due to metabolism involving CYP2C19 gene polymorphisms in the liver. In this study, the second-line PPI switching strategy to overcome CYP2C19 polymorphism gave a benefit in improving the eradication rate. Although 
there was no statistical difference, the simple second-line PPI switching effect showed a better eradication rate. The efficacy of PPIs could differ among individuals depending on genetic polymorphisms in the hepatic drug-metabolizing CYP2C19 enzyme, even though the impact of rabeprazole was less significant. Rabeprazole is primarily metabolized through a non-enzymatic reduction to a thioether derivative, and the CYP2C19 enzyme is partly involved in its metabolism. ${ }^{24,25}$ There are several studies on PPI-related differences in acid suppression potency according to CYP2C19 polymorphisms, and most of them pertained to first-line triple therapy. Until now, different researchers have reported different results, and there is no clear consensus. ${ }^{17,18,26}$ A previous meta-analysis showed that only rabeprazole had a higher eradication rate in PMs, and the efficacy of various PPIs seemed to be similar when they were used for $H$. pylori eradication in standard triple therapy. ${ }^{15}$ In other meta-analyses, there were no statistically significant differences in the eradication rates between patients with two CYP2C19 genotypes taking lansoprazole or rabeprazole-based therapy. ${ }^{13,14}$ In second-line treatments, there is one well-designed study comparing the efficacy of rabeprazole for eradication therapy. ${ }^{27}$ This Taiwanese study of 190 patients reported that CYP2C19 polymorphisms affected the success of quadruple therapy, and rabeprazole-based second-line quadruple therapy had a better eradication rate than esomeprazole-based therapy. The strength of our study would be its relatively large scale, including 479 patients who failed first-line standard triple therapy, although there was no direct polymorphism analysis of the enrolled patients.

Even though the effects of the various PPIs were not confirmed in first-line standard triple therapy, an influence of CYP2C19 polymorphism could affect the eradication rate in second-line rescue regimens. Previous studies revealed that tailored therapy based on PCR analysis of CYP2C19 polymorphism and clarithromycin resistance (A2143G and A2142G) could reach ideal eradication rates. $^{28-30}$

However, this therapy might be an economic burden to the patients in the aspects of cost-effectiveness and time involvement. Instead of PCR techniques, the use of strategies to overcome the effects of CYP2C19 polymorphism could greatly benefit the public health of large populations.

There were several limitations to our study. First, this study was a retrospective review conducted at a single academic center. Second, we did not analyze all data over 10 years. Because we did not use all kinds of PPIs, including second-generation PPIs, until 2012, we could analyze the whole data to evaluate only the efficacy of PPI switching.

In conclusion, in order to improve the successful second-line $H$. pylori eradication rate, which is gradually decreasing, PPI switching could be effective and strategies using esomeprazole and rabeprazole, which are not influenced significantly by CYP2C19 genetic polymorphism, are recommended after the failure of standard triple therapy for $H$. pylori infections.

\section{CONFLICT OF INTEREST}

No potential conflict of interest relevant to this article was reported.

\section{ORCID}

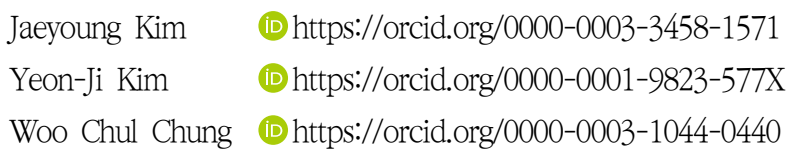

\section{REFERENCES}

1. Mentis A, Lehours P, Mégraud F. Epidemiology and diagnosis of Helicobacter pylori infection. Helicobacter 2015;20 Suppl $1: 1-7$.

2. Malfertheiner P, Leodolter A, Peitz U. Cure of Helicobacter pylori-associated ulcer disease through eradication. Baillieres Best Pract Res Clin Gastroenterol 2000;14:119-132.

3. Fennerty MB, Lieberman DA, Vakil N, Magaret N, Faigel DO, Helfand M. Effectiveness of Helicobacter pylori therapies in a clinical practice setting. Arch Intern Med 1999;159:1562-1566.

4. Choi IJ, Kook MC, Kim YI, et al. Helicobacter pylori therapy for the prevention of metachronous gastric cancer. N Engl J Med 2018;378:1085-1095.

5. Uno Y. Prevention of gastric cancer by Helicobacter pylorieradication: a review from Japan. Cancer Med 2019;8:3992-4000.

6. Liou JM, Lee YC, El-Omar EM, Wu MS. Efficacy and long-term safety of $H$. pylori eradication for gastric cancer prevention. 
Cancers (Basel) 2019;11:E593.

7. Drossman DA. Functional gastrointestinal disorders: history, pathophysiology, clinical features and Rome IV. Gastroenterology 2016;150:1262-1279.e2.

8. Sugano K, Tack J, Kuipers EJ, et al. Kyoto global consensus report on Helicobacter pylori gastritis. Gut 2015;64:1353-1367.

9. Malfertheiner P, Megraud F, O'Morain CA, et al. Management of Helicobacter pylori infection--the Maastricht IV/Florence Consensus Report. Gut 2012;61:646-664.

10. Marin AC, McNicholl AG, Gisbert JP. A review of rescue regimens after clarithromycin-containing triple therapy failure (for Helicobacter pylori eradication). Expert Opin Pharmacother 2013;14:843-861.

11. Tomalik-Scharte D, Lazar A, Fuhr U, Kirchheiner J. The clinical role of genetic polymorphisms in drug-metabolizing enzymes. Pharmacogenomics J 2008;8:4-15.

12. Hagymási K, Müllner K, Herszényi L, Tulassay Z. Update on the pharmaco genomics of proton pump inhibitors. Pharmacogenomics 2011;12:873-888.

13. Padol S, Yuan Y, Thabane M, Padol IT, Hunt RH. The effect of CYP2C19 polymorphisms on $H$. pylori eradication rate in dual and triple first-line PPI therapies: a meta-analysis. Am J Gastroenterol 2006;101:1467-1475.

14. Zhao F, Wang J, Yang Y, et al. Effect of CYP2C19 genetic polymorphisms on the efficacy of proton pump inhibitor-based triple therapy for Helicobacter pylori eradication: a meta-analysis. Helicobacter 2008;13:532-541.

15. Vergara M, Vallve M, Gisbert JP, Calvet X. Meta-analysis: comparative efficacy of different proton-pump inhibitors in triple therapy for Helicobacter pylori eradication. Aliment Pharmacol Ther 2003;18:647-654.

16. Dojo M, Azuma T, Saito T, Ohtani M, Muramatsu A, Kuriyama M. Effects of CYP2C19 gene polymorphism on cure rates for Helicobacter pylori infection by triple therapy with proton pump inhibitor (omeprazole or rabeprazole), amoxycillin and clarithromycin in Japan. Dig Liver Dis 2001;33:671-675.

17. Kuo CH, Lu CY, Shih HY, et al. CYP2C19 polymorphism influences Helicobacter pylori eradication. World J Gastroenterol 2014;20:16029-16036.

18. Jørgensen SF, Reims HM, Frydenlund D, et al. A cross-sectional study of the prevalence of gastrointestinal symptoms and path- ology in patients with common variable immunodeficiency. Am J Gastroenterol 2016;111:1467-1475.

19. Choi HS, Park DI, Hwang SJ, et al. Double-dose, new-generation proton pump inhibitors do not improve Helicobacter pylori eradication rate. Helicobacter 2007;12:638-642.

20. Labenz J. Current role of acid suppressants in Helicobacter pylori eradication therapy. Best Pract Res Clin Gastroenterol 2001;15:413-431.

21. Chiba T, Malfertheiner P, Satoh H. Proton pump inhibitors: a balanced view. Basel: Karger Publishers, 2013.

22. Marcus EA, Inatomi N, Nagami GT, Sachs G, Scott DR. The effects of varying acidity on Helicobacter pylori growth and the bactericidal efficacy of ampicillin. Aliment Pharmacol Ther 2012;36:972-979.

23. Sanaka M, Yamamoto T, Kuyama Y. Effects of proton pump inhibitors on gastric emptying: a systematic review. Dig Dis Sci 2010;55:2431-2440.

24. Sharara AI. Rabeprazole: the role of proton pump inhibitors in Helicobacter pylori eradication. Expert Rev Anti Infect Ther 2005;3:863-870.

25. Horn J. Review article: relationship between the metabolism and efficacy of proton pump inhibitors--focus on rabeprazole. Aliment Pharmacol Ther 2004;20 Suppl 6:11-19.

26. Miwa H, Misawa H, Yamada T, Nagahara A, Ohtaka K, Sato N. Clarithromycin resistance, but not CYP2C-19 polymorphism, has a major impact on treatment success in 7-day treatment regimen for cure of $H$. pylori infection: a multiple logistic regression analysis. Dig Dis Sci 2001;46:2445-2450.

27. Kuo CH, Wang SS, Hsu WH, et al. Rabeprazole can overcome the impact of CYP2C19 polymorphism on quadruple therapy. Helicobacter 2010;15:265-272.

28. De Francesco V, Margiotta M, Zullo A, et al. Clarithromycin-resistant genotypes and eradication of Helicobacter pylori. Ann Intern Med 2006;144:94-100.

29. Francavilla R, Lionetti E, Castellaneta S, et al. Clarithromycin-resistant genotypes and eradication of Helicobacter pylori. J Pediatr 2010;157:228-232.

30. Woo HY, Park DI, Park H, et al. Dual-priming oligonucleotide-based multiplex PCR for the detection of Helicobacter pyloriand determination of clarithromycin resistance with gastric biopsy specimens. Helicobacter 2009;14:22-28. 